\title{
Resuscitation with intraosseous lines in neonatal units
}

\author{
A W R Kelsall
}

\begin{abstract}
Two infants on the neonatal unit aged 143 days and 47 days with difficult venous access were successfully resuscitated from episodes of collapse using the intraosseous route.
\end{abstract}

(Arch Dis Child 1993;68:324-325)

Intraosseous resuscitation of babies and infants is taught to residents in North America in the paediatric advanced life support course. ${ }^{1}$ No such formal training is widely available in Great Britain. Residents are encouraged to site intraosseous lines in an arrest if conventional venous access has not been established within one minute. The chosen route is in to the medial tibial plate (figure). The needle is simply inserted using a screwing action at right angles to the bone surface, when the marrow cavity is entered the needle gives. Correctly positioned, the needle is very stable and fluid can be infused without resistance.

\section{Case reports}

Recently two babies on our neonatal unit have collapsed and venous access was impossible. Both had had multiple peripheral cannulations and previously attempted central catheterisation.

CASE 1

A boy born at 27 weeks' gestation, weighing $595 \mathrm{~g}$, was transferred at 130 days of age for repair of a massive inguinal hernia. He had been reventilated because of bronchopul-

Neonatal Unit, Rosie Maternity Hospital, Cambridge CB2 2QQ

Correspondence to: Dr Kelsall.

Accepted 28 October 1992

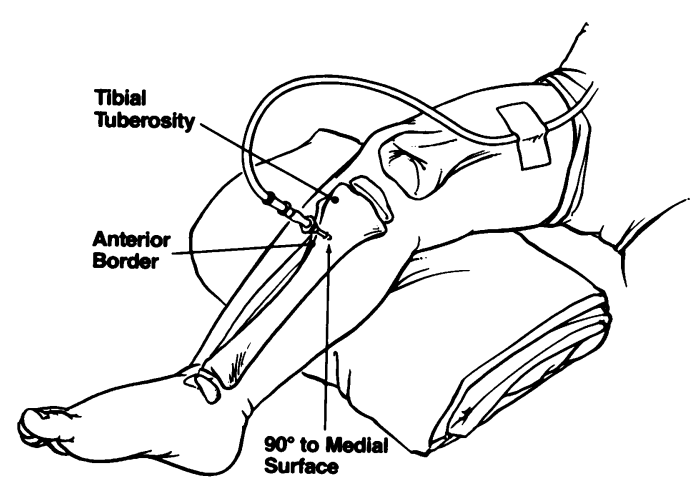

Intraosseous cannulation technique. Reproduced with permission from the Textbook of Pediatric Advanced Life Support, 1988. Copyright American Heart Association. monary dysplasia and infection, which was being treated with antibiotics, diuretics, and a course of dexamethasone. Herniorrhaphy was performed at 137 days. He failed a trial of extubation after four days. Venous access was a continuing problem; central venous access had been unsuccessful. On day 144, weighing $1920 \mathrm{~g}$, his wound dehisced and small bowel spilled into the incubator. $\mathrm{He}$ was pale, bradycardic, hypotensive, and poorly perfused and venous access was impossible. Intraosseous access was established into the right tibial plate using a $22 \mathrm{G} \times 1.5$ inch spinal needle. He was rapidly resuscitated with $80 \mathrm{ml}$ of $4.5 \%$ human albumin solution given as $20 \mathrm{ml}$ bolus pushes. After restoration of the circulating volume conventional peripheral venous and arterial lines were inserted. The wound was repaired. During surgery a central line was inserted allowing removal of the intraosseous line an hour later. However, the child deteriorated progressively, developing respiratory failure, further wound dehiscence, and continuing problems with venous access. $\mathrm{He}$ died at 155 days. No complications with the intraosseous insertion site were observed.

\section{CASE 2}

A baby with Beckwith-Wiedemann syndrome was born at 34 weeks' gestation weighing $3760 \mathrm{~g}$. He was transferred aged 6 days, with necrotising enterocolitis and respiratory distress syndrome requiring ventilation. Normoglycaemia was maintained with infusions of $15-20 \%$ dextrose, via a right subclavian venous line inserted on day 6 . Perforation of the caecum necessitated resection of the ascending colon. Postoperatively he had episodes of hypoglycaemia, bilateral lower lobe atelectasis, and continuing problems with venous access. At 41 days, weighing $4.6 \mathrm{~kg}$ his fourth central line failed. He was not tolerating enteral feeds and his blood glucose was $2 \cdot 1 \mathrm{mmol} / 1$. He was tachycardic, dyspneoic, pale, and unresponsive. An experienced paediatric anaesthetist was unable to establish central or peripheral venous access and thus a $22 \mathrm{G}$ spinal needle was inserted into the right tibial plate. A total of $120 \mathrm{ml}$ of $4.5 \%$ human albumin solution was rapidly infused to restore the circulating blood volume. $\mathrm{He}$ was electively ventilated. A $10 \%$ dextrose infusion was commenced and the infant was taken to theatre one hour later, with normal blood sugars and good perfusion for the surgical insertion of a Hickman line. The intraosseous line was removed on return 
to the unit. He made steady progress and was discharged home four months later. There were no problems with the leg when he was reviewed at eight months.

\section{Discussion}

Intraosseous lines are widely used in North American emergency rooms to achieve venous access in the critically sick child. The lines are sited for short periods only, and are removed once standard cannulas have been placed. The morbidity of this procedure is low. ${ }^{2}$ The American Academy of Pediatrics recommend that the technique is used up to the age of 3 years. Despite its widespread use, most recently reported by Ryder et $a l^{3}{ }^{3}$ a specific role in the neonatal unit has not been described. With the increased survival of very premature babies, many of whom have major problems with venous access, situations will arise when a shocked baby can only be resuscitated using central routes. In the two babies described in this report, despite experienced people in attendance neither central nor peripheral venous access was possible. The ability to site intraosseous lines allowed fluid resuscitation and stabilisation of these critically ill infants, after which, conventional venous access was established. Neither child appeared to develop any problems around the infusion site.

Heinild et al presented their experience of almost 1000 bone marrow infusions. ${ }^{2}$ Complications occurred with hypertonic infusions of $50 \%$ dextrose or after any prolonged infusions over several days. Fluid extravasation was noted at the time of insertion. Infection usually became apparent within a week of the infusion. Berg reported that a wide variety of drugs and fluids could be safely administered via the marrow cavity. ${ }^{4}$

Insertion of a spinal needle into the tibia provides a simple, safe, and effective route to stabilise critically ill infants. Providing the line is in situ only for a few hours and very hypertonic fluids are avoided the risks are minimal. The technique should be taught to all neonatal medical staff.

AWRK is funded by Action Research.

1 Chameides $\mathrm{L}$, ed. Textbook of pediatric advanced life support. Dallas: American Heart Association and American Academy of Pediatrics, 1988:43-4.

2 Heinild S, Soderguard T, Tudvad F. Bone marrow infusions in childhood: experiences from a thousand infusions. 7 Pediatr 1947;30:400-11.

3 Ryder IG, Munro HM, Doull IJM. Intraosseous infusion for resuscitation. Arch Dis Child 1991;66:1442-3.

4 Berg RA. Emergency infusion of catecholamines into bone marrow. Am $\mathcal{F}$ Dis Child 1984;138:810. 